\title{
Investigative Modeling of Symmetric Fiber Bragg Grating as Dispersion Compensation for Optical Transmission System
}

\author{
A. A. Shabaneh ${ }^{1 *}$ \\ 1. Department of Telecommunication Technology Engineering, Faculty of Engineering and Technology, \\ Palestine Technical University-Kadoorie (PTUK), Tulkarm, Palestine.
}

${ }^{(*)}$ E-mail: a.shabaneh@ptuk.edu.ps

Received: 25/10/2020 Accepted: 26/12/2020

DOI: 10.7149/OPA.53.4.51052

\begin{abstract}
:
Dispersion is a serious issue related to the integrity of data transmission in high-speed optical fiber communication systems. Fiber Bragg gratings (FBGs) are among the most important components used to reduce dispersion in these systems. FBGs are simple, and have low-cost filter for wavelength selection, low insertion loss and customized reflection spectrum, and wide bandwidth. This study investigates the optical transmission system performance of a symmetric FBG for dispersion compensation. Transmission scheme simulation is analyzed with different parameters based on an OptiSystem simulator. Symmetric FBG and avalanche photodetector are used to enhance the evaluation of an optical transmission system. Optical fiber length, attenuation coefficient, input power, bit rate, and FBG grating length parameters are also investigated in detail to determine system optimization. The developed simulation design demonstrated high gain, low noise figure, substantial output power, and high Q-Factor for long transmission distance of up to $60 \mathrm{~km}$ with 0.2 $\mathrm{dB} / \mathrm{km}$ attenuation coefficient.
\end{abstract}

Keywords: Dispersion compensation, Symmetric Fiber Bragg Grating (FBG), Optisystem simulator, Photodiode APD, Reflected signal.

\section{REFERENCES AND LINKS}

[1] B. P. B. M. A. Parida, "Fiber Bragg grating as a dispersion compensator in an optical transmission system using optisystem software," 2015.

[2] A. Bhardwaj and G. Soni, "Performance analysis of 20Gbps optical transmission system using fiber Bragg grating," Int J Sci Res Publ, vol. 5, pp. 1-4, 2015.

[3] M. Othman et al., "An analysis of $10 \mathrm{Gbits} / \mathrm{s}$ optical transmission system using fiber Bragg grating (FBG)," IOSR Journal of Engineering, vol. 2, no. 07, pp. 55-61, 2012.

[4] P. Arasu et al., "Absorbance properties of gold coated fiber Bragg grating sensor for aqueous ethanol," Journal of the European Optical Society-Rapid publications, vol. 9, 2014.

[5] S. Mohammadi, S. Mozaffari, and M. M. Shahidi, "Simulation of a transmission system to compensate dispersion in an optical fiber by chirp gratings," International Journal of Physical Sciences, vol. 6, no. 32, pp. 7354-7360, 2011.

[6] M. Morin, "Fiber Bragg Grating interferometers for chromatic dispersion compensation," ed: Google Patents, 2004.

[7] N. M. Faiyaz, A. I. Omi, and M. Faisal, "Optimization of apodization profile of chirped fiber Bragg grating for chromatic dispersion compensation: dispersion compensation using chirped apodized FBG," in 2014 International Conference on Electrical Engineering and Information \& Communication Technology, 2014: IEEE, pp. 1-5. 
[8] H. A. Mahmood, "DCF with FBG for dispersion compensation in optical fiber link at various bit rates using duobinary modulation format," Engineering and Technology Journal, vol. 36, no. 5 Part (A) Engineering, pp. 514-519, 2018.

[9] H. A. Mahmood and R. K. Ahmed, "Performance evaluation of high data rate optical communication system utilizing FBG compensated dispersion schemes under different modulation techniques," Diyala Journal of Engineering Sciences, vol. 10, no. 2, pp. 94-106, 2017.

[10] F. Pelletier and A. V. Van, "Adjustable chromatic dispersion compensator," ed: Google Patents, 2004.

[11] A. A. Khadir, B. F. Dhahir, and X. Fu, "Achieving optical fiber communication experiments by optisystem," International Journal of Computer Science and Mobile Computing, vol. 3, no. 6, pp. 4253, 2014.

[12] M. Chakkour, O. Aghzout, B. Ait Ahmed, F. Chaoui, and M. El Yakhloufi, "Chromatic dispersion compensation effect performance enhancements using FBG and EDFA-wavelength division multiplexing optical transmission system," International Journal of Optics, vol. 2017, 2017.

[13] D. Meena and M. Meena, "Design and analysis of novel dispersion compensating model with chirp fiber bragg grating for long-Haul transmission system," in Optical and Wireless Technologies: Springer, 2020, pp. 29-36.

[14] R. Mishra and R. Bharti, "An analysis of $10 \mathrm{Gbits} / \mathrm{S}$ Optical transmission system using Fiber Bragg Grating (FBG) and double EDFA," International Journal of Advanced Research in Computer Science, vol. 8, no. 8, 2017.

[15] H. Hamadouche, B. Merabet, and M. Bouregaa, "Performance Comparison of APD and PIN Photodiodes using RZ and NRZ."

[16] O. Kharraz and D. Forsyth, "Performance comparisons between PIN and APD photodetectors for use in optical communication systems," Optik, vol. 124, no. 13, pp. 1493-1498, 2013.

[17] A. Soni and M. Gupta, "Analysis and Design of Optimized Fractional Order Low Pass Bessel Filter," Journal of Circuits, Systems and Computers, 2020.

[18] P. Xia, L.-H. Zhang, and Y. Lin, "Simulation Study of Dispersion Compensation in Optical Communication Systems Based on Optisystem," in Journal of Physics: Conference Series, 2019, vol. 1187, no. 4: IOP Publishing, p. 042011.

[19] M. Singh and S. Arora, "Performance Comparison between PIN and APD Photodiodes for use in Free Space Optical Communication Link," IJIRAE, vol. 2, no. 8, 2015.

\section{Introduction}

At present, among the main cores of recent telecommunication systems is optical fiber because it has a wide range of applications, rapid development, and involves a high degree of complexity. Optical fibers carry signals from one point to another in the form of light, without any electricity in the transmission medium [1]. Fiber optic scheme contains three central components: a transmitter device that converts electrical signal to light signal, an optical fiber cable that transfers light in the core surrounded by cladding, and a receiver that admits the light signal and converts it back to an electrical signal $[2,3]$.

During transmission, signal power is decreased because of attenuation. To solve this problem, optical networks allow the application of erbium doped fiber amplifier (EDFA) with fiber Bragg grating (FBG) [4]. Since the invention of FBG in the 1980s, FBG has been extensively applied in existing telecommunication infrastructures, and predicted to play an important role in next generation of networks and internets. FBGs have several applications to enhance the quality and diminish the costs in optical networks [5, 6]. FBGs are simple, and have low-cost filter for wavelength selection and low insertion loss, customized reflection spectrum, and wide bandwidth. Moreover, FBGs are simply inclusion of alternative modulation of refractive index (RI) in the optical fiber core. RI of FBGs can be changed with the spatial distribution of light intensity through certain doping mode; moreover, the RI period changes, similar to Bragg grating [7-10]. FBGs are applied to improve transmission performance. The use of FBGs in communication system aims to solve the chromatic dispersion problem owing to its low cost and negligible effects. Moreover, FBGs are commonly chosen as significant components to compensate for dispersion in optical communication systems. The use of FBGs reduces the linewidth, which results in sending extremely narrow pulses; hence, dispersion is reduced and Q-factor is increased [5-10]. 
In a single mode fiber (SMF), chromatic dispersion is a phenomenon generated because of the dependency of group index $(\mathrm{Ng})$ on wavelength. Thus, such a dependence creates a time extension in broadcasted pulses. Frequency is increased along the pulse once the dispersion coefficient factor of the fiber is negative. After a specific distance, the extension of pulses leads to significant errors at the receiver, thereby leading to missing information. The dispersion properties of materials interact with optical transmission systems. Thereby increasing or decreasing the total pulse dispersion as the signal propagates. This outcome is a reference to the chirpings of FBGs. Furthermore, EDFA is utilized to address the attenuation effects. However, applying EDFA is proposed to compensate for loses in optical communication systems $[5,11]$.

Chakkour et al. [12] proposed an optical transmission system using FBGs and EDFA to overcome the chromatic dispersion and attenuation phenomena. They investigated the optical fiber length $(5 \mathrm{~km}$ to 30 $\mathrm{km}$ ) and attenuation coefficient parameters in detail to deal with the optimized corresponding parameter values. Their results indicated that the performance of the optimized design parameters is extremely efficient in terms of output power (dBm), noise figure (dB), gain (dB), and Q-Factor. The best performance parameters of the proposed model were given in terms of Q-Factor, gain, noise figure, and output power are $50,14.42 \mathrm{~dB}, 8.08 \mathrm{~dB}$, and $12.20 \mathrm{dBm}$, respectively. Therefore, the choice of chirped FBGs as dispersion compensation is a considerably suitable technique, and their parameters can be adapted to obtain optimized and superior results in terms of gain and Q-Factor in the designed optical link.

This study investigates and discusses a new model of symmetric FBGs as dispersion compensation for optical transmission system utilizing Optisystem. Different parameter settings are applied and analyzed. In addition, eye diagrams, values of Q-Factor, gain, noise figure, and signal output power are investigated for the entire system.

\section{Simulation Details}

\section{2. a. FBGs}

FBGs have alternating modulation of RI, which acts as a wavelength selective mirror with low-cost filtering. Furthermore, FBGs show high immunity toward varying ambient circumstances and electromagnetic (EM) radiation. FBGs are easy to fabricate and have small sizes. Fig. 1 shows the principle operation of the FBG light with the curve spectra of the input, transmitted, and reflected signals $[12,13]$.

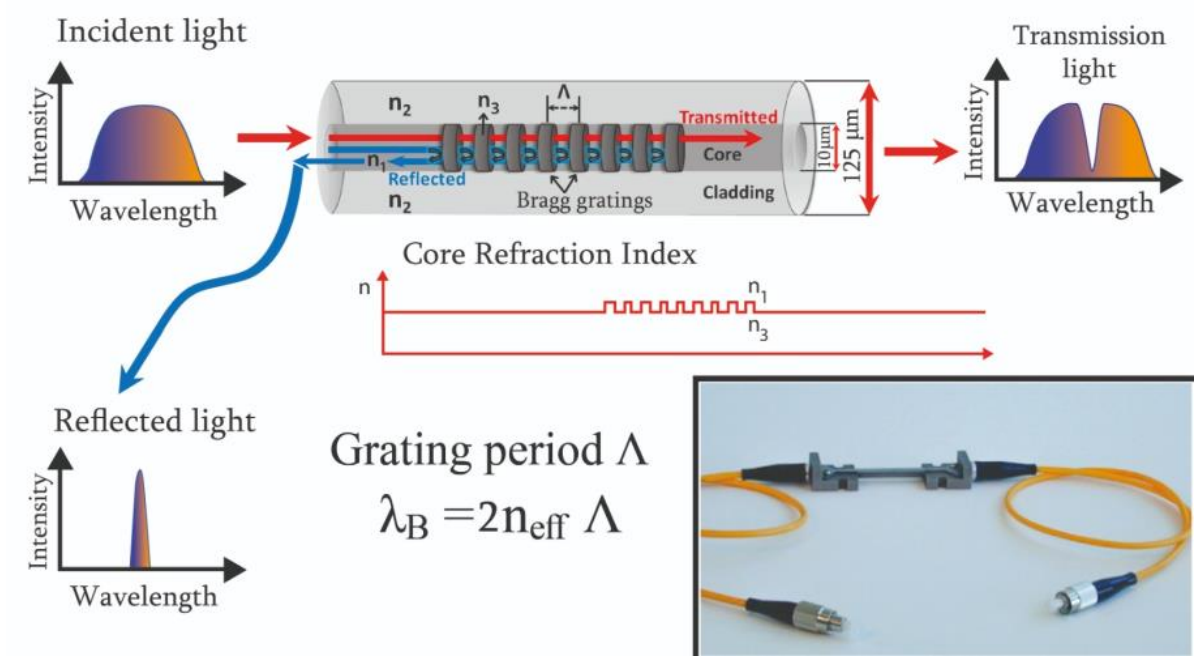

Fig. 1. Principle operation of the FBG light.

Given that FBGs are germanium doped, they are sensitive to strong argon ion laser radiation. Subsequently, numerous techniques are used to produce gratings in optical fibers, in which widespread types of pulsed and continuous lasers are used in the visible and ultraviolet regions. The grating distance or period of gratings determines the wavelength that will be reflected. This wavelength is called the Bragg wavelength and its relationship with the grating period is presented as follows: 


$$
\lambda_{\mathrm{B}}=2 \mathrm{n}_{\mathrm{eff}} \Lambda \text {, }
$$

where the Bragg grating wavelength $\lambda_{B}$ is the free space wavelength of the input light reflected from the grating, $n_{\text {eff }}$ is the effective RI of the fiber core at the free space center wavelength, and $\Lambda$ is the grating period of FBG.

A uniform grating can be expressed as sinusoidal modulation of the fiber core RI as follows:

$$
\mathrm{n}(\mathrm{z})=\mathrm{n}_{\mathrm{core}}+\delta \mathrm{n}[1+\cos (2 \pi \mathrm{z} / \Lambda+\varphi(\mathrm{z}))]
$$

where $\mathrm{n}_{\text {core }}$ is the core RI when it is not radiated and $\delta \mathrm{n}$ is the amplitude of the induced RI differences.

In optical transmission systems, FBGs work as appropriate compensator for chromatic dispersion for communication link. FBGs are a single mode fiber which discloses the core to the periodic pattern of intensive ultraviolet light. The disclosure increases RI, which is permanently increased. The disclosure pattern previously creates a fixed index modulation called grating. After periodic refraction is changed, a small reflected light is produced. Thereafter, a small reflected light is combined into a large reflected light at a certain wavelength. This wavelength is called Bragg's wavelength, precisely when the grating period is nearly half the input light's wavelength. The remainder of the light is transparent $[1,12,14]$.

\section{2. b. Description of Components}

Pseudo-random bit sequence (PRBS) generator is used to create a continuous data signal in the form of bit rates. The setup mode controls the algorithm applied to produce the bit sequence probability: random number creator is applied through parameter sign probability of ones in the sequence. Order: a sequence of ones is generated. Zeros: a sequence of zeros is generated. One of the most significant advantages of a non-return-zero (NRZ) pulse generator is controlling bandwidth. Owing to this feature of the NRZ pulse generator, returning signals to zero between bits are not wasting the data signal bandwidth. Moreover, the NRZ pulse generator is used in producing electrical data signal for the modulation process. When voltage varies, it has the capability to set the bits in a state. Hence, specifying where the bits would start and stop becomes simple $[1-3,5,12]$.

Continues wave (CW) laser diode is used to create optical signals that provide input signal with $1550 \mathrm{~nm}$ wavelength and input power of $1,5,10,15,20$, and $25 \mathrm{dBm}$, which are externally modulated at 10, 15, 20, 30, and $40 \mathrm{Gbits} / \mathrm{s}$. In addition, the CW laser is connected with a NRZ pseudorandom binary sequence in a Mach-Zehnder (M-Z) modulator with $7 \mathrm{~dB}$ of extinction ratio. Mach-Zehnder modulator is an optical modulator constructed on the fundamental of interferometry. This modulator is applied for electrically controlling the output amplitude or phase of the light wave passing through the device. Accordingly, the output signal is modulated according to voltage $[1-3,5,12]$. Furthermore, high-speed laser mostly adopts the external modulation mode, which includes the $\mathrm{M}-\mathrm{Z}$ waveguide modulator and electric absorption modulator. The M-Z modulator is typically composed of a lithium niobate $\left(\mathrm{LiNO}_{3}\right)$ material and distributed feedback laser (DFB), which has good chirp elimination. Therefore, the M-Z modulator is appropriate for the long-distance transmission of high-speed systems.

SMF within a small transparent core radius is applied to enable the propagation of one mode light surrounded by a transparent cladding material with low RI of refraction. Light is passed at the core by the total internal reflection mechanism. This system is appropriate for a long distance communications transmission with high-speed data rate with low attenuation compared with electrical transmission lines. SMF acts as a carrier of waveguide signal to transmit light between the two ends of the fiber. The range of fiber length is from $25 \mathrm{~km}$ to $100 \mathrm{~km}$ for transmitting data signal. Symmetric FBGs are applied to compensate for the chromatic dispersion of common fibers. FBGs are a part of a common single mode fiber that is similar to grating. Optical amplification EDFA is utilized to compensate for and overcome losses and to amplify signal before being received by the photodetector APD at the receiver part of an optical transmission system. EDFA has superior characteristics, such as high gains, bandwidths, low noises, and high efficiencies $[1-3,5,12]$.

Avalanche photodetector (APD photodiode) is utilized to detect light (photons) at the receiver by converting light directly to electric current. One photon yields one electron. Numerous electron-hole pairs 
are created owing to the influence of ionization. APD is similar to a P-N photodiode or PIN photodiode, in which electron-hole pairs are created because of the absorption of photons. However, the fundamental impact of ionization increases the magnitude of the photocurrent at APD photodetector compared to PIN photodiode. Consequently, the APD photodetector can detect extremely weak (low-level) signal owing to high current-gain bandwidth product. Lastly, a properly designed silicon APD photodetector can provide a response time of approximately $1 \mathrm{~ns}$. The difference with the PIN diode is that the absorption of a photon of incident light sets off an electron-hole pair avalanche breakdown, thereby creating up to 100 additional electron-hole pairs. This property makes the APD photodiode more sensitive than the PIN photodiode $[15,16]$.

Low pass Bessel filter is designed to offer maximum flat group delay and maximum linear phase change across the band and into the transition region. In addition, this type of filter approximates Gaussian type filters. The function of low pass Bessel filter is to sacrifice sharpness in the magnitude response [17].

\section{2. c. Optisystem Simulator}

Optisystem software is an advanced optical communication construction simulator. This software is set for designing, optimizing, and testing any category of optical link in the physical layer of the broad spectrum for optical networks. Optical networks are from long-haul schemes to local area networks (LANs), apart from metropolitan area networks (MANs). In addition, optical networks may expand with the addition of user elements and simple interfaces to a range of broadly used library tools. Optical networks are also based on the realistic modeling of optical fiber communication systems, a powerful novel simulation environment, and a truly hierarchical description of devices and layouts. It can support operators to design experiments and simulate many applications, such as CATV or WDM/TDM or networks, dispersion map plans, transmitters, receivers, and amplifier designs [1-3, 5, 12].

\section{2. d. Simulation Model and Design Consideration}

The simulation model is operated with a basic optical communication, which consists of a transmitter, optical fiber link, and receiver in the Optisystem software, as shown in Fig. 2. The scheme transmits data using optical carrier wave from transmitter to receiver via optical fiber cable. Input signal contains electrical data represented by $0 \mathrm{~s}$ and $1 \mathrm{~s}$ generated by an NRZ pseudorandom binary sequence. Thereafter, the input signal is modulated with a semiconductor laser, which is represented via continuous wave laser through the M-Z modulator. CW laser supports input signal with $1550 \mathrm{~nm}$ wavelength and input power of $5 \mathrm{dBm}$, and externally modulated at 10,15, 20, 30 and $40 \mathrm{Gbits} / \mathrm{s}$ with an NRZ pseudorandom binary sequence in the $\mathrm{M}-\mathrm{Z}$ modulator with $7 \mathrm{~dB}$ of extinction ratio. The optical fiber applied is a single mode fiber because it provides high data rate, has low dispersion, and suitable for long-haul distance, making fit for use as a transmission link cable.

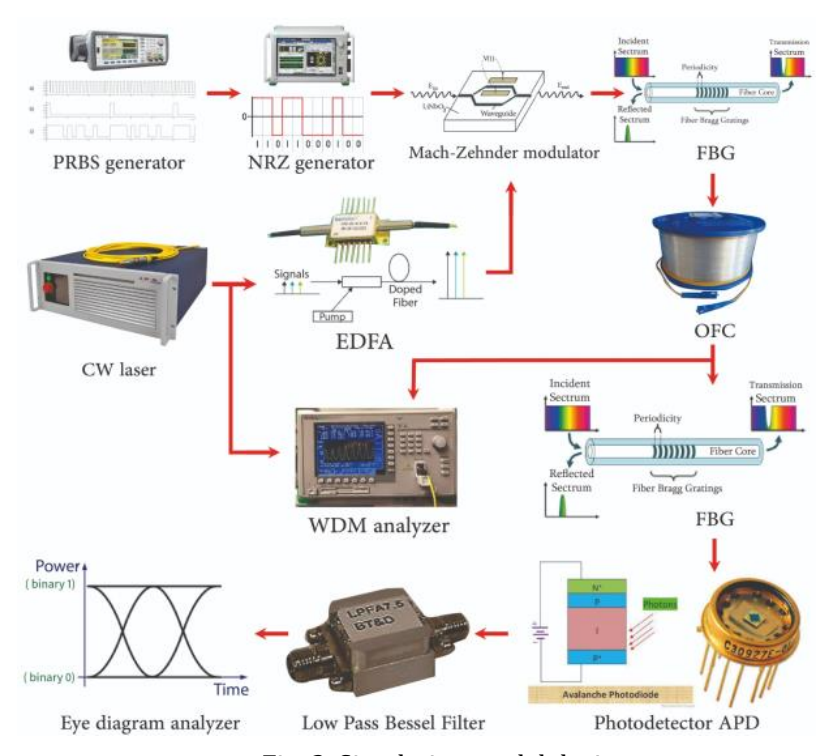

Fig. 2. Simulation model design. 
Table 1 shows the settings of the global simulation parameters selected for the simulation model.

TABLE 1. Simulation parameter settings

\begin{tabular}{|c|c|}
\hline Parameters & Values \\
\hline Length of fiber $(\mathrm{km})$ & 5 \\
\hline $\begin{array}{l}\text { Attenuation index } \\
(\mathrm{dB} / \mathrm{km})\end{array}$ & 0.2 \\
\hline $\begin{array}{l}\text { Dispersion } \\
(\mathrm{ps} / \mathrm{km} / \mathrm{nm})\end{array}$ & 16.75 \\
\hline $\begin{array}{l}\text { Dispersion slope } \\
\left(\mathrm{ps} / \mathrm{nm}^{2} / \mathrm{km}\right)\end{array}$ & 0.075 \\
\hline $\begin{array}{c}\text { Reference } \\
\text { wavelength }(\mathrm{nm})\end{array}$ & 1550 \\
\hline $\begin{array}{l}\text { Differential group } \\
\text { delay (ps/km) }\end{array}$ & 0.2 \\
\hline $\begin{array}{l}\text { C/W input power } \\
(\mathrm{dBm})\end{array}$ & 5 \\
\hline EDFA length $(\mathrm{km})$ & 5 \\
\hline
\end{tabular}

Symmetric FBGs are applied as a dispersion chromatic compensator. The grating length (step size) varies from $4 \mathrm{~mm}$ to $8 \mathrm{~mm}$. However, $4 \mathrm{~mm}$ is the most appropriate length for the proposed model, which matches with the $1550-\mathrm{nm}$ light source wavelength.

The simulation is conducted by placing symmetric FBGs along the path before and after OFC and without using FBGs. Table 2 shows the global simulation parameters selected for the symmetric FBGs.

TABLE 2. Simulation parameter settings for the symmetric FBGs

\begin{tabular}{|c|c|}
\hline \hline Parameters & Values \\
\hline \hline Frequency (THz) & 193.1 \\
Dispersion (ps/nm) & 125 \\
Effective index \\
Apodization \\
function \\
$\begin{array}{c}\text { Tanh parameter } \\
\text { Chirp function }\end{array}$ \\
$\begin{array}{c}\text { Linear parameter } \\
(\mu \mathrm{GH})\end{array}$ \\
$\begin{array}{c}\text { Length (mm) } \\
\text { Linear }\end{array}$ \\
\hline \hline
\end{tabular}

\section{Results and Analysis}

The simulation and investigation of the layout is designed using Optisystem 7.0 simulation software. The novelty of the developed simulation design, which applies symmetric FBGs before and after OFC, indicates high performance and ideal evaluation in terms of gain and NF. The eye diagrams and gain (dB), noise figure $(\mathrm{dB})$, signal output power $(\mathrm{dBm})$, and Q-Factor are tabulated and tested for varying values of optical fiber length $(\mathrm{km})$, attenuation coefficient $(\mathrm{dB} / \mathrm{km})$, input power $(\mathrm{dBm})$, bit rate $(\mathrm{G}$ Bits $/ \mathrm{s})$, and FBG grating 
length (mm). Fig. 3 illustrates the main design layout of FBGs as symmetric dispersion compensation for the optical transmission system.

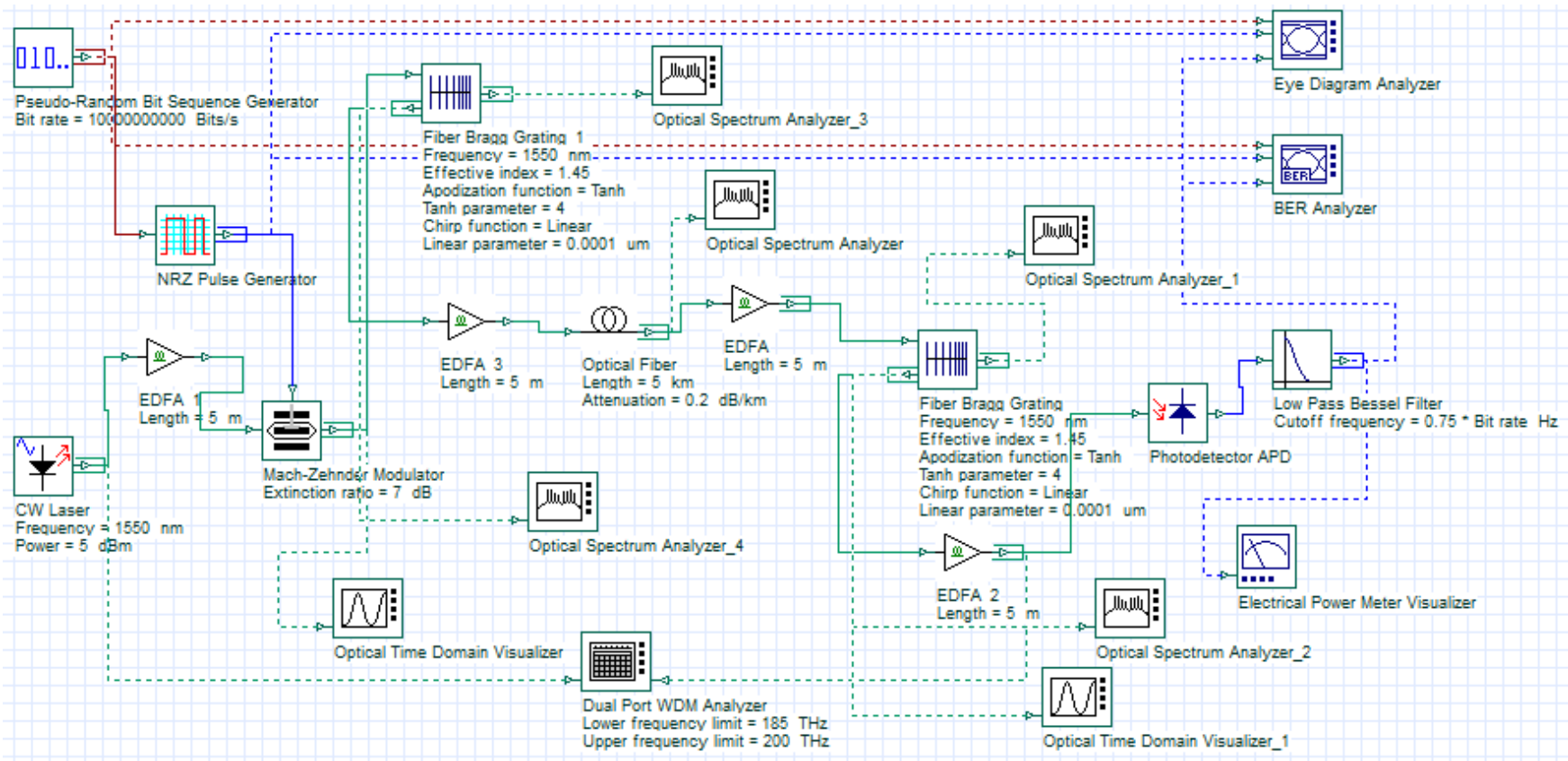

Fig. 3. Designed model of the symmetric FBGs.

\section{3. a. Output results of the simulated model with and without FBGs}

Initially, the designed layout is simulated without obtaining dispersion compensation, waveforms, and eye diagrams. Thereafter, FBGs are set before and after OFC to simulate symmetric compensation. The simulation process indicates that under the condition of system setting parameters, the eye diagram opening condition and dispersion compensation effect are analysed, in which the symmetrical compensation performance is the best.

Fig. 4 demonstrates the eye diagram, optical spectrum, and optical time domain before using the symmetric FBGs. Fig. 5 shows the eye diagram, optical spectra before and after using symmetric FBGs, and the optical time domain after applying symmetric FBGs. An optical spectrum analyzer (OSA) is used to monitor the output signals after each component. Moreover, OSA is used as a tool to measure optical power as a function of wavelength. The spectrum image indicates that FBGs function as a light filter, as shown in Fig 5. In addition, Fig. 4 shows that the eye diagram is not clear and not completely open, thereby leading to low Q-Factor value. Meanwhile, Fig. 5 shows a clear and open eye diagram, which leads to high Q-Factor value. The red line represents the curve of the minimum bit error rate (BER) for the design without FBGs and for the developed design, as illustrated in Figs. 4 and 5.

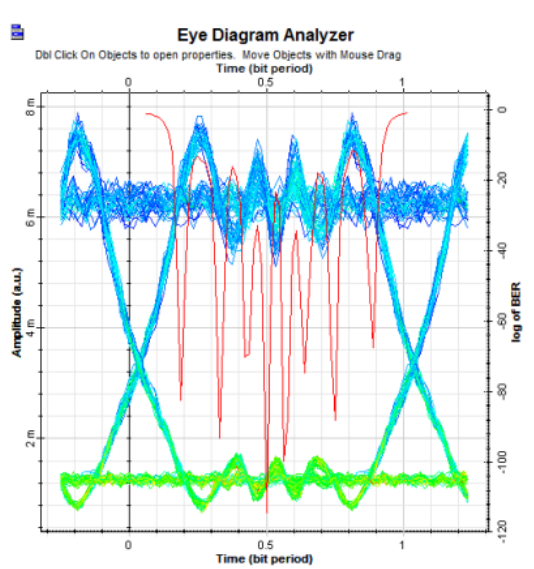

뭅

Optical Spectrum Analyzer

를 Optical Time Domain Visualizer Dbl Click On Objects to open properties. Move Objects with Mouse Drag Db Click On Objects to open properties. Move Objects with Mouse Drag
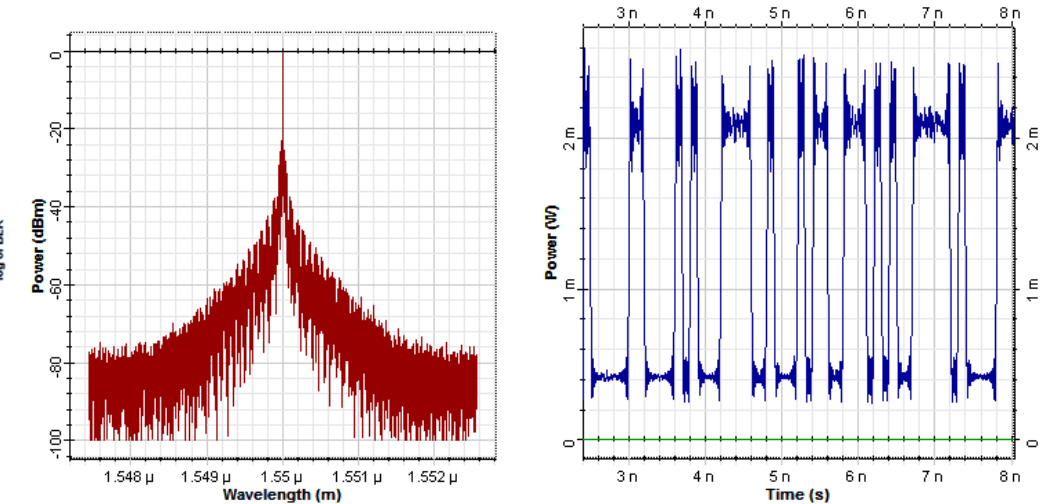

Fig. 4. Eye diagram and optical spectrum before applying the symmetric FBGs. 


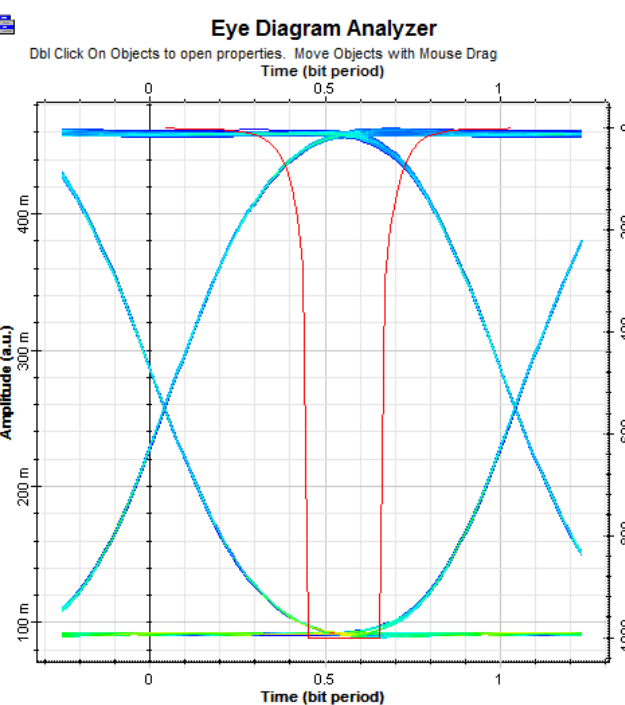

量

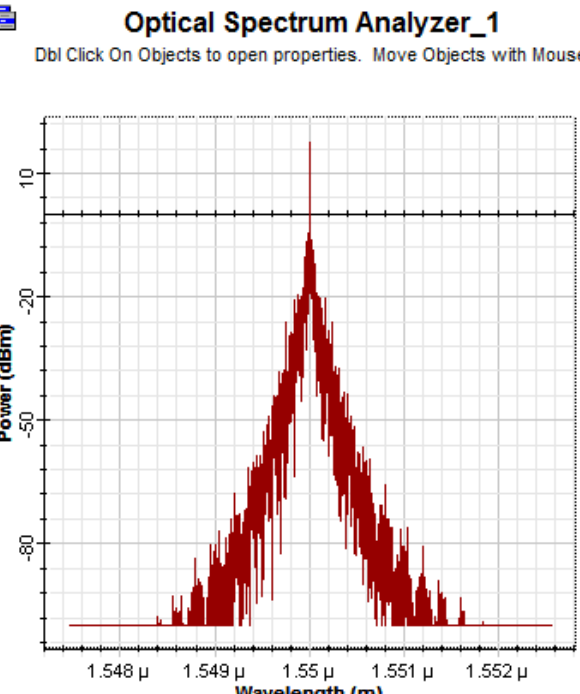

를 Optical Spectrum Analyzer_3 Dbl Click On Objects to open properties. Move Objects with Mouse Drag

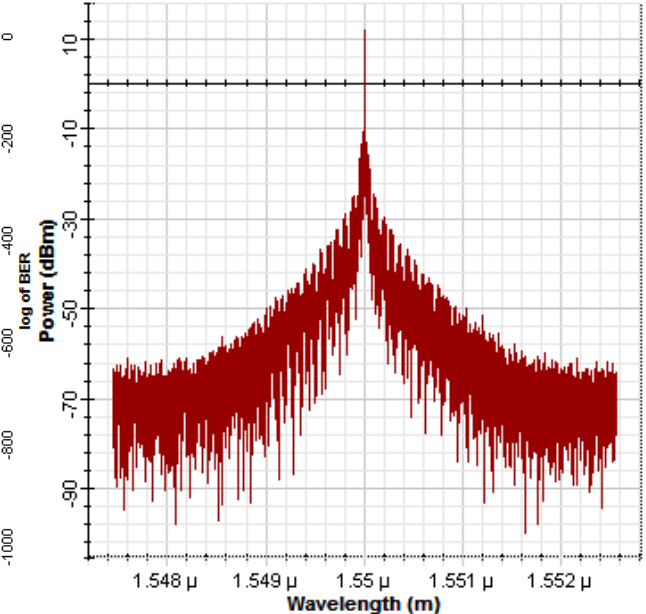

를 Optical Time Domain Visualizer_1

Dbl Click On Objects to open properties. Move Objects with Mouse Drag

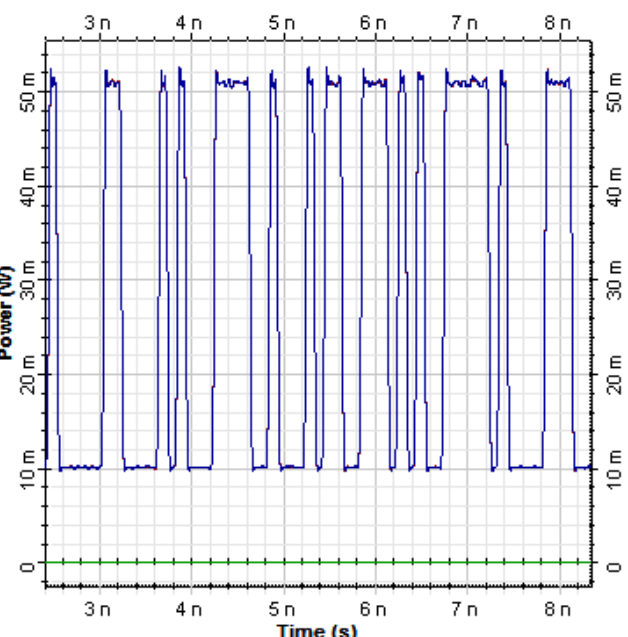

Fig. 5. Eye diagram and optical spectrum after applying the symmetric FBGs.

\section{3. b. Output results of the simulated model by modifying the SMF cable lengths}

The performance effect of varying the optical fiber length for optical transmission system is investigated. Fig. 6 indicates the eye diagrams for the different OFC length values. A comparison among the six figures (eye diagrams) indicates that when the OFC length increases from $5 \mathrm{~km}$ to $20 \mathrm{~km}$, the eye closure remains nearly the same (clear and open). Nonetheless, when the OFC lengths are 30,50, and $60 \mathrm{~km}$, the eye diagrams become unclear and the Q-Factor values degrade substantially, as shown in Table 3. Thus, the efficiency of the optical network transmission is enhanced and developed in this study for different OFC lengths. The current research considers Q-Factor to calculate the transmission system performance of the received signal. The Q-Factor equation is expressed as follows:

$$
Q=f_{r} / \Delta f,
$$

where $f_{r}$ and $\Delta f$ are the resonant frequency and resonance width or full width at half maximum (FWHM), respectively. 

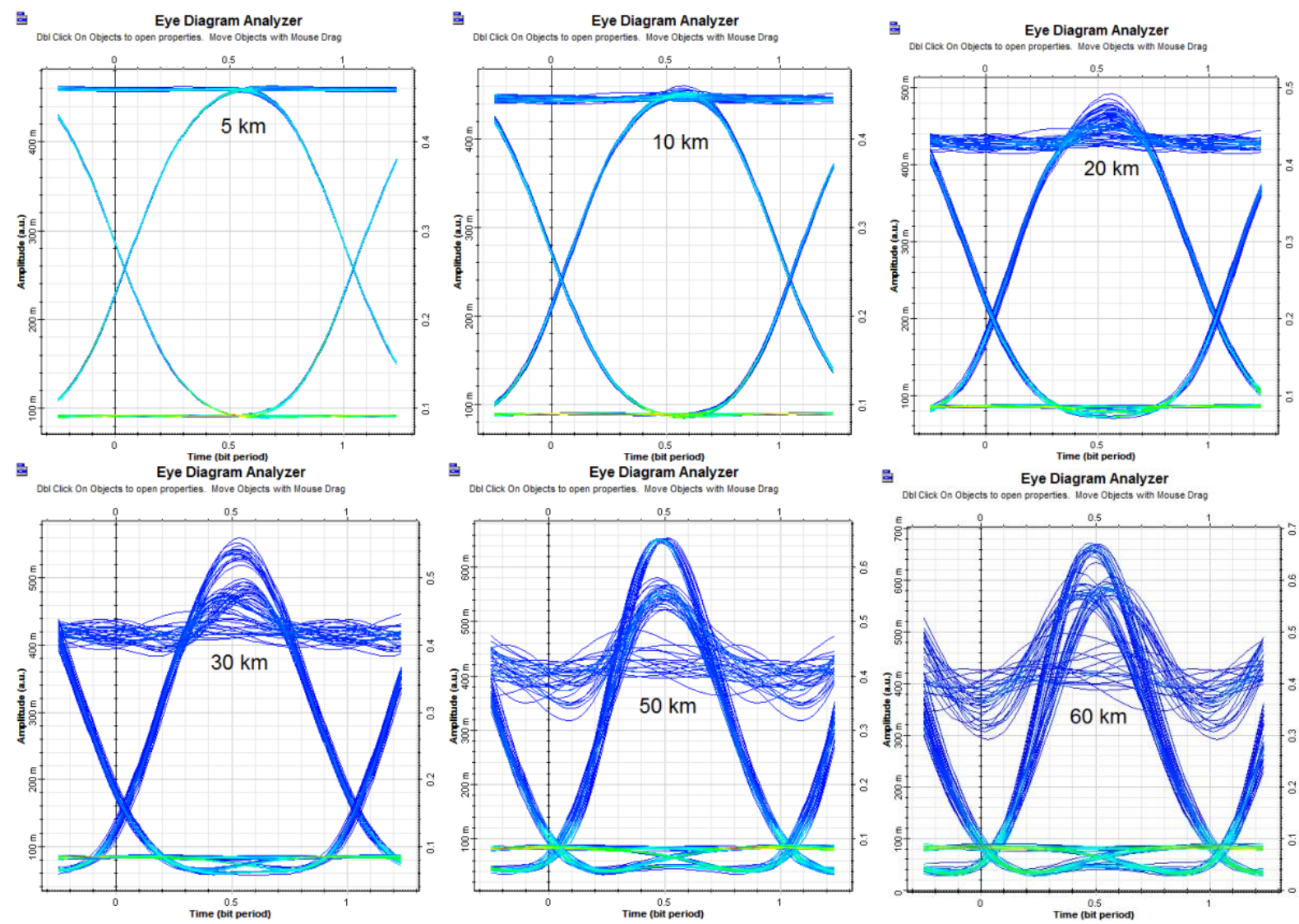

Fig. 6. Eye diagrams for the different OFC length values.

Table 3 shows the results of the output readings at different OFC length values. Evidently, small values are observed in the gain, signal output power, and Q-Factor, which decrease as the OFC length increases. By contrast, the noise figure increases gradually as fiber length increases.

TABLE 3. Output results by varying the OFC lengths

\begin{tabular}{|c|c|c|c|c|}
\hline \hline $\begin{array}{c}\text { OFC Length } \\
(\mathrm{km})\end{array}$ & Gain (dB) & NF (dB) & $\begin{array}{c}\text { Output signal } \\
(\mathrm{dBm})\end{array}$ & Q-Factor \\
\hline \hline 5 & 14.37 & 6.80 & 19.37 & 223 \\
10 & 14.19 & 6.93 & 19.18 & 104 \\
20 & 13.84 & 7.28 & 18.84 & 46 \\
30 & 13.55 & 7.67 & 18.55 & 18 \\
50 & 13.04 & 8.64 & 18.03 & 8 \\
60 & 12.92 & 9.10 & 17.92 & 5 \\
\hline \hline
\end{tabular}

\section{3. c. Output results of the simulated model by modifying the attenuation coefficients}

This study analyzes the impact of attenuation coefficient on the performance of the simulated optical transmission system. Accordingly, the loss is expressed in $\mathrm{dB} / \mathrm{km}$. Moreover, the loss introduced via the propagation down of a fiber is provided as follows:

$$
\alpha=-10 \log _{10}\left(P_{\text {out }} / P_{\text {in }}\right),
$$

where $P_{\text {out }}$ and $P_{\text {in }}$ are the output power of the signal and input power in fiber, respectively. 
By contrast, the eye diagram opening is evidently high at $0.2,1$, and $3 \mathrm{~dB} / \mathrm{km}$, as shown in Fig. 7 .
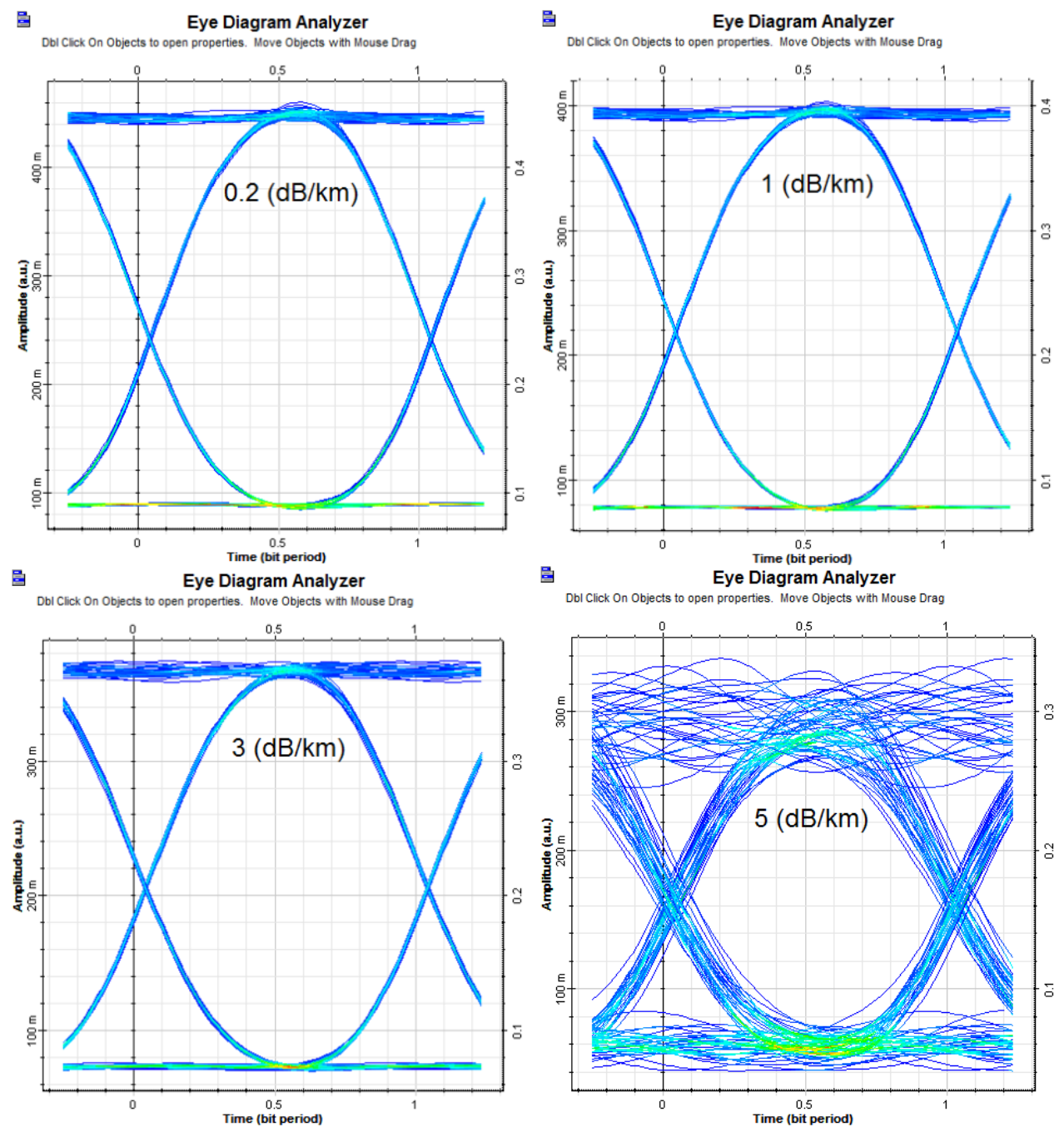

Fig. 7. Eye diagrams for the different attenuation coefficient values.

The lowest value of NF and the highest value of gain, output power, and Q-Factor are at $0.2 \mathrm{~dB} / \mathrm{km}$. Thus, $0.2 \mathrm{~dB} / \mathrm{km}$ attenuation coefficient is the most suitable value applied to the proposed designed model.

Table 4 presents the results of the output readings at different attenuation coefficient values. As shown in Table 4, attenuation coefficient increases and a slight decrease is recorded of gain. In addition, the signal output power and Q-Factor are decreased. As a result, the NF increases hugely as the attenuation coefficient increases.

TABLE 4. Output results by varying the attenuation coefficients

\begin{tabular}{|c|c|c|c|c|}
\hline \hline $\begin{array}{c}\text { Attenuation } \\
\text { coefficients } \\
(\mathrm{dB} / \mathrm{km})\end{array}$ & Gain $(\mathrm{dB})$ & $\mathrm{NF}(\mathrm{dB})$ & $\begin{array}{c}\text { Output signal } \\
(\mathrm{dBm})\end{array}$ & Q-Factor \\
\hline \hline 0.2 & 14.37 & 6.80 & 19.37 & 223 \\
1 & 13.94 & 7.14 & 19.93 & 216 \\
3 & 13.63 & 9.00 & 18.62 & 191 \\
5 & 13.53 & 15.19 & 18.52 & 135 \\
\hline
\end{tabular}




\section{3. d. Output results of the simulated model by modifying the bit rates}

Fig. 8 shows three eye diagrams for varying the values of bit rate in the pseudo-random bit sequence generator of 10, 20 and 30 (G Bits/s). A clear open eye is obtained for 10-G Bits/s bit rate. However, unclear eye diagrams are shown for values of $20 \mathrm{G}$ Bits/s and $30 \mathrm{G}$ Bits/s. These values are verified and reflected clearly with Q-Factor, as presented in Table 6.
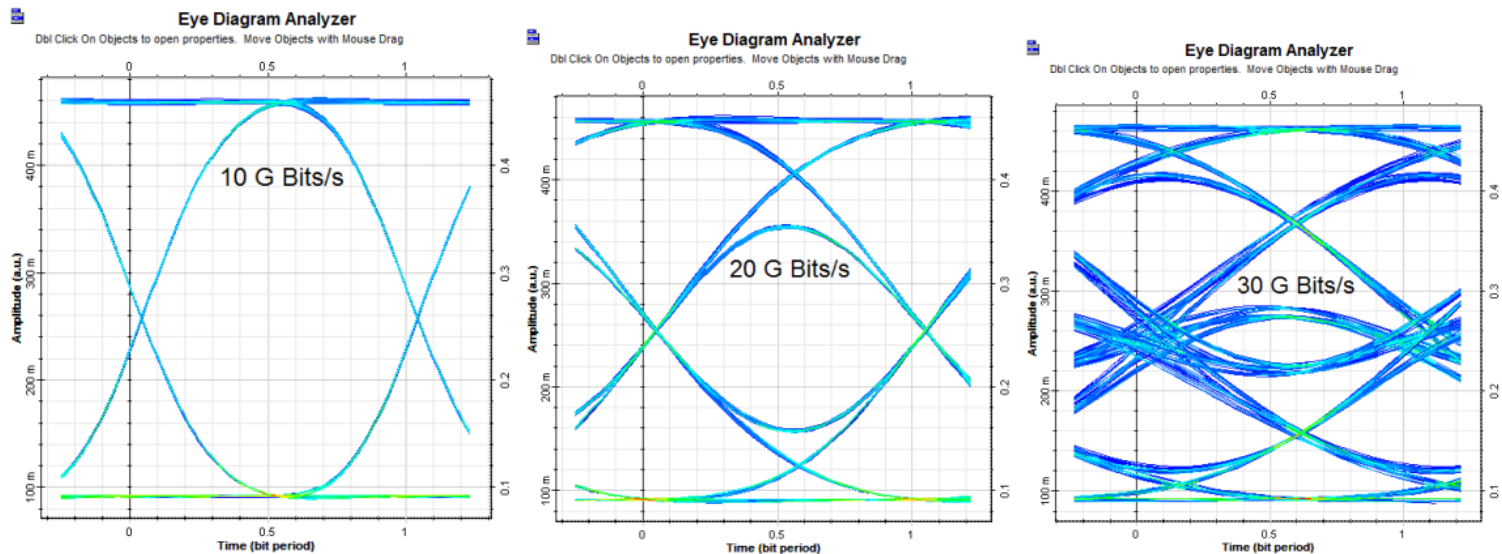

Fig. 8. Eye diagrams for the different bit rate values.

A small change in values of gain, NF, and output power are shown in Table 5. However, Q-Factor is vastly decreased through varying bit rates. Table 6 presents the results of the output readings at different values of input power. As input power increases, a considerable change in value of gain is evident. Moreover, a slight increase is observed in NF, signal output power, and Q-Factor as input power increases.

TABLE 5. Output results by varying the bit rates

\begin{tabular}{|c|c|c|c|c|}
\hline \hline $\begin{array}{c}\text { Input power } \\
(\mathrm{dBm})\end{array}$ & Gain (dB) & NF (dB) & $\begin{array}{c}\text { Output signal } \\
(\mathrm{dBm})\end{array}$ & Q-Factor \\
\hline \hline 1 & 18.37 & 5.05 & 19.36 & 206 \\
5 & 14.37 & 6.80 & 19.37 & 223 \\
10 & 9.38 & 9.99 & 19.38 & 234 \\
15 & 4.41 & 13.73 & 19.41 & 241 \\
20 & -0.49 & 17.41 & 19.50 & 250 \\
25 & -5.24 & 20.86 & 19.75 & 262 \\
\hline \hline
\end{tabular}

TABLE 6. Output results via varying the input power

\begin{tabular}{|c|c|c|c|c|}
\hline \hline $\begin{array}{c}\text { Bit rate } \\
\text { (G Bits/s) }\end{array}$ & Gain (dB) & NF (dB) & $\begin{array}{c}\text { Output signal } \\
(\mathrm{dBm})\end{array}$ & Q-Factor \\
\hline \hline 10 & 14.37 & 6.80 & 19.37 & 223 \\
15 & 14.29 & 6.93 & 19.28 & 12 \\
20 & 14.23 & 6.95 & 19.23 & 4 \\
30 & 14.20 & 7.03 & 19.19 & 2 \\
40 & 14.19 & 7.08 & 19.18 & 0 \\
\hline \hline
\end{tabular}

\section{3. e. Output results of the simulated model by modifying the FBG lengths}

Fig. 9 shows the eye diagrams by varying the symmetric FBG lengths in $\mathrm{mm}$. The eye diagrams are open and clear for 4, 6 and $8 \mathrm{~mm}$ of the symmetric FBG lengths. 

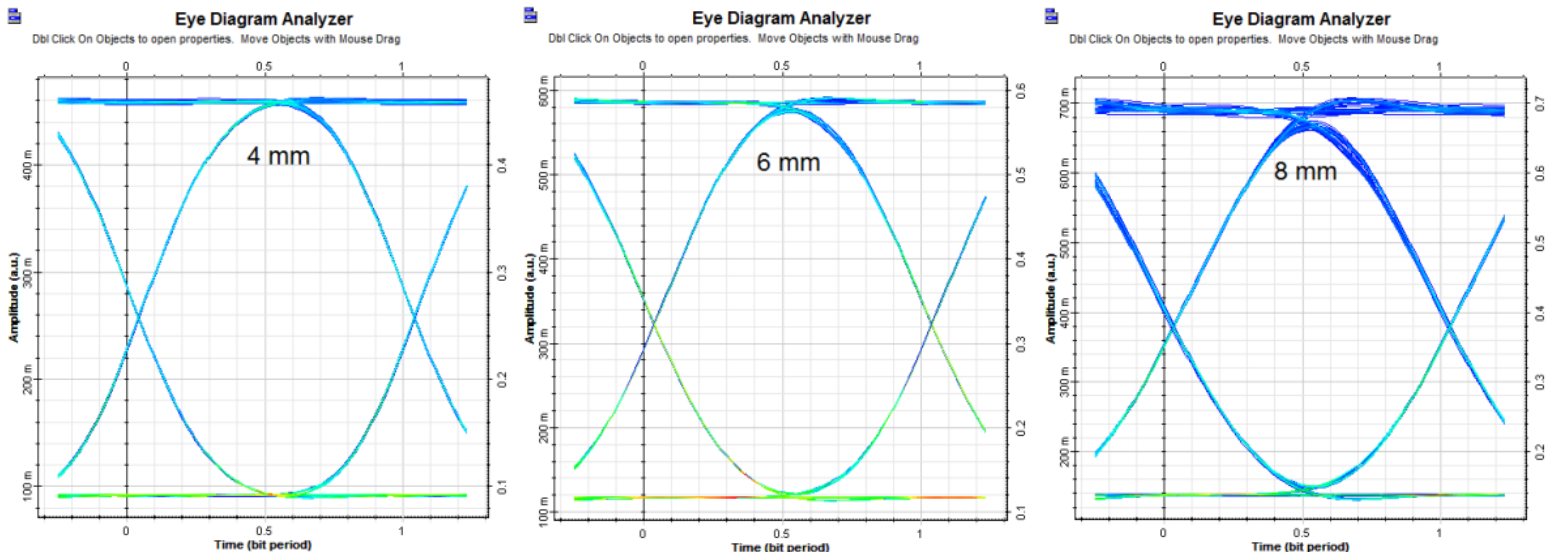

Fig. 9. Eye diagrams for the different FBG length values.

Table 7 shows the different values of the FBG grating length. Consequently, gain and output power increase as the FBG length increases. Conversely, NF and Q-Factor decrease as the FBG length increases.

TABLE 7. Output results via varying the FBG length

\begin{tabular}{|c|c|c|c|c|}
\hline \hline $\begin{array}{c}\text { FBG grating } \\
\text { length }(\mathrm{mm})\end{array}$ & Gain $(\mathrm{dB})$ & NF $(\mathrm{dB})$ & $\begin{array}{c}\text { Output signal } \\
(\mathrm{dBm})\end{array}$ & Q-Factor \\
\hline \hline 4 & 14.37 & 6.80 & 19.37 & 223 \\
5 & 14.93 & 6.14 & 19.93 & 142 \\
6 & 15.41 & 5.64 & 20.40 & 81 \\
7 & 15.79 & 5.24 & 20.79 & 56 \\
8 & 16.11 & 4.94 & 21.10 & 37 \\
\hline
\end{tabular}

Table 8 presents a comparison summary between the proposed model without FBG, without EDFA, and with symmetric FBG with two EDFA. Furthermore, this study simulates a new developed model based on two FBG (before and after OFC), two EDFA, and APD photodetector, and compares with those of M. Chakkour et al. and P. Xia et al. $[12,18]$. From the comparison, the current study shows high and stable performances with low NF along the communication system. Table 8 also shows that the proposed model presents several advantages in terms of gain, signal output power, total power, BER, Q-Factor, and signal quality performance with low NF. In general, the simulation design is characterized by an excellent gain performance with $14.37 \mathrm{~dB}$, NF of $6.80 \mathrm{~dB}$, signal output power of $19.37 \mathrm{dBm}$, and Q-Factor of 223. The developed design shows high gain and clear eye diagram with 223 Q-Factor compared with 50.27, 190 and 23.82 in Chakkour et al. [12], P. Xia et al. [18], and Bhardwaj et al. [2], respectively. The proposed design shows high signal output power of $19.37 \mathrm{dBm}$ compared with Chakkour et al. [12] at $12.20 \mathrm{dBm}$.

TABLE 8. Comparison parameters with the proposed model

\begin{tabular}{|c|c|c|c|c|c|}
\hline \hline Parameters & $\begin{array}{c}\text { Without FBG } \\
\text { + without } \\
\text { EDFA }\end{array}$ & $\begin{array}{c}\text { With FBG } \\
\text { after OFC+ } \\
\text { without EDFA }\end{array}$ & $\begin{array}{c}\text { With FBG } \\
\text { before and } \\
\text { after OFC } \\
\text { without EDFA }\end{array}$ & $\begin{array}{c}\text { With FBG and } \\
\text { EDFA after } \\
\text { OFC }\end{array}$ & $\begin{array}{c}\text { With } \\
\text { symmetric } \\
\text { FBG + two } \\
\text { EDFA before } \\
\text { and after OFC }\end{array}$ \\
\hline \hline Gain (dB) & -4.21 & 7.23 & 12.59 & 13.96 & 14.37 \\
NF (dB) & 4.21 & 6.89 & 8.65 & 6.48 & 6.80 \\
Signal output \\
power (dBm) \\
$\begin{array}{c}\text { Q-Factor } \\
\text { Total power }\end{array}$ & -17.778 & 12.22 & 17.59 & 18.96 & 19.37 \\
(dBm) & 6.13 & 17.18 & 16.33 & 19.14 & 223 \\
BER & $6.10981 \mathrm{e}^{-115}$ & 0 & 0 & 0 & 19.78 \\
\hline \hline
\end{tabular}


Fig. 10 shows the curve relationship among gain, NF, and output power at different OFC lengths. Evidently, the smallest value of NF and the best value of gain and output power are at $0.2 \mathrm{~dB} / \mathrm{km}$ and symmetric FBG length of $6 \mathrm{~mm}$ before and after OFC.

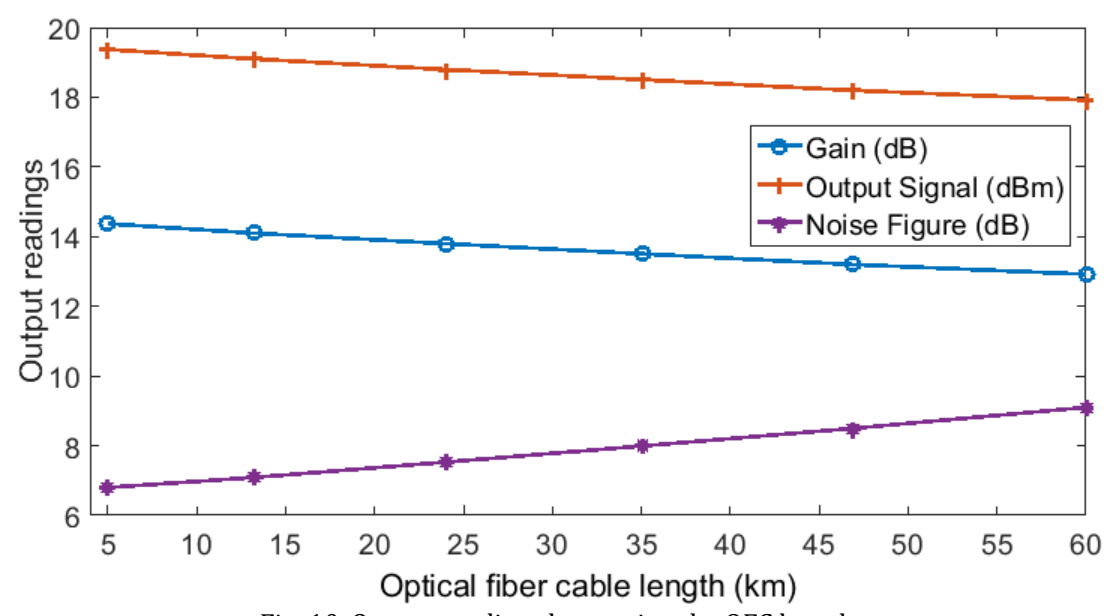

Fig. 10. Output readings by varying the OFC length.

Lastly, Table 9 shows a comparison between using APD and PIN photodetector at the receiver side. The comparison was done for $5 \mathrm{~km}$ of $\mathrm{OFC}$ with 0.2 attenuation coefficient and $5 \mathrm{dBm}$ input power of the CW laser. Table 9 also presents that utilizing the APD photodetector yields higher system performance compared with PIN photodetector in terms of output noise power, output signal power, output total power, Q-Factor, and gain. The output readings of this analysis indicate that the values of Q-Factor and output signal power are at maximum when APD is used as the receiving photodiode at $1550 \mathrm{~nm}$ wavelength. APD creates a higher signal-to-noise ratio (SNR) than PIN at equal optical power source. This enhancement in SNR is the result of the APD gain [1].

TABLE 9. Comparison parameter readings between the APD and PIN photodetectors

\begin{tabular}{|c|c|c|c|c|c|c|}
\hline \hline Device/Parameter & $\begin{array}{c}\text { Output } \\
\text { noise } \\
\text { power } \\
(\mathrm{dBm})\end{array}$ & $\begin{array}{c}\text { Output signal } \\
\text { power }(\mathrm{dBm})\end{array}$ & $\begin{array}{c}\text { Output total } \\
\text { power } \\
(\mathrm{dBm})\end{array}$ & Q-Factor & Gain $(\mathrm{dB})$ & $\mathrm{NF}(\mathrm{dB})$ \\
\hline \hline $\begin{array}{c}\text { With APD } \\
\text { photodetector } \\
\begin{array}{c}\text { With PIN } \\
\text { photodetector }\end{array}\end{array}$ & -36.80 & 19.37 & 19.78 & 223 & 14.37 & 6.80 \\
\hline \hline
\end{tabular}

\section{Conclusions}

Symmetric FBGs as chromatic dispersion compensation for optical transmission system is successfully developed and investigated in this study. The proposed model with different parameters is simulated via Optisystem software to overcome dispersion and signal attenuation. The novel design of symmetric FBGs (before and after OFC) demonstrates high efficiency on the received signal quality and low noise ratio with markedly satisfactory performances. Consequently, the FBG length and input power are directly proportional to the signal power. In addition, the OFC length and attenuation coefficient are directly proportional to NF. However, gain decreases when the OFC length increases. The developed model shows high gain, low NF, and stable responses. The simulation design is characterized by an excellent gain performance with $14.37 \mathrm{~dB}$, NF of $6.80 \mathrm{~dB}$, signal output power of $19.37 \mathrm{dBm}$, and Q-Factor of 223 . Consequently, the simulation performance of the proposed optical system indicates its potential in longdistance transmission, such as telephone signals and Internet communication usages.

\section{Acknowledgments}

The author would like to acknowledge Palestine Technical University-Kadoorie (PTUK) for supporting and funding this research. 